FACTA UNIVERSITATIS (NIŠ)

Ser. Math. Inform. Vol. 34, No 4 (2019), 709-727

https://doi.org/10.22190/FUMI1904709A

\title{
FUZZY SUBSETS OF THE PHENOTYPES OF $F_{2}$-OFFSPRING
}

\author{
Madeleine Al-Tahan and Bijan Davvaz
}

(C) 2019 by University of Niš, Serbia | Creative Commons Licence: CC BY-NC-ND

\begin{abstract}
This paper presents a connection between fuzzy sets, biological inheritance and hyperstructures in which we consider the set of phenotypes of the second generation $F_{2}$ in different types of inheritance, define fuzzy subsets of it and construct a sequence of join spaces associated to each of its types.
\end{abstract}

Keywords. Hyperstructures; fuzzy subsets; join spaces; hypergroups; automata theory.

\section{Introduction}

The Hyperstructure theory was introduced in 1934, at the eighth Congress of Scandinavian Mathematicians, when F. Marty [17] defined hypergroups as natural generalization of the concept of group based on the notion of hyperoperation, analyzed their properties and applied them to groups. In the following decades and nowadays, a number of different hyperstructures are widely studied from the theoretical point of view and for their applications to many subjects of pure and applied mathematics: geometry, topology, cryptography and code theory, graphs and hypergraphs, probability theory, binary relations, theory of fuzzy and rough sets, automata theory, economy, etc. (see $[4,6,7]$ ). A hypergroup is an algebraic structure similar to a group, but the composition of two elements is a non-empty set. One of motivations for the study of hyperstructures comes from biological inheritance. In [10], M. Ghadiri and B. Davvaz used the concept of $H_{v}$-semigroup structure in the $F_{2}$-genotypes with cross operation and proved that it is an $H_{v^{-}}$ semigroup and they determined the kinds of the $H_{v}$-subsemigroups of $F_{2}$-genotypes (see also [7]). Another motivation for the study of hyperstructures comes from physical phenomenon as the nuclear fission. This motivation and the results were presented by S. Hošková, J. Chvalina and P. Račková (see [13], [14]). In [9], the

Received March 17, 2019; accepted August 14, 2019

2010 Mathematics Subject Classification. Primary 20N20;; Secondary 92D10, 03E72 
authors provided, for the first time, a physical example of hyperstructures associated with the elementary particle physics, Leptons. They have considered this important group of the elementary particles and shown that this set along with the interactions between its members can be described by the algebraic hyperstructures. On the other hand, the concept of fuzzy sets has been introduced by L. A. Zadeh in 1965 (see [29]) as an extension of the classical notion of set, when he proposed the idea of a multi-valued logic, which extends the traditional concept of a bivalent logic, which becomes a particular case of the new theory. The fuzzy set theory is based on the principle called by L. A. Zadeh "the principle of incompatibility", that is "the closer a phenomenon is studied, the more indistinct its definition becomes". Fuzzy sets are sets whose elements have degrees of membership. In classical set theory, the membership of elements in a set is assessed in binary terms according to a bivalent condition an element either belongs or does not belong to the set. By contrast, fuzzy set theory permits the gradual assessment of the membership of elements in a set; this is described with the aid of a membership function valued in the real unit interval $[0,1]$. Fuzzy sets generalize classical sets, since the indicator functions of classical sets are special cases of the membership functions of fuzzy sets, if the latter only take values 0 or 1 .

The early theories of heredity were those of Greek scientists (Hippocrates and Aristotle); their theories were similar to Darwin's later ideas on Pangenesis. The latter states that the whole of parental organisms participate in heredity while adapting to cell theory. Much of Darwin's model was speculatively based on inheritance of tiny heredity particles that could be transmitted from parent to offspring [5]. The hypothesis was eventually replaced by Mendel's laws of inheritance where Gregor Mendel first traced patterns of certain traits in pea plants and showed that they obeyed certain statistical rules. Scientific studies of Mendelian inheritance began in 1866 with the experiments of Mendel, the founder of modern genetics [18]. Mendel worked out the mathematical rules for the inheritance of characteristics in the garden pea. The significance of his discovery was not recognized until 1900, when three botanists: Hugo de Vries, Carl Correns and Erich von Tschermak began independently conducting similar experiments with plants and arrived at conclusions similar to those of Mendel. Coming across Mendel's paper, they interpreted their results in accord with his principles and drew attention to his pioneering work. And by 1915 the basic principles of Mendelian genetics had been applied to a wide variety of organisms. Mendel discovered the principles of heredity by crossing different varieties of pea plants and analyzing the transmission pattern of traits in subsequent generations. He began by studying monohybrid crosses, those between parents that differed in a single characteristic. Mendel's approach to the study of heredity was effective for several reasons. The foremost was his choice of an experimental subject, the pea plant, Pisum sativum, which offered obvious advantages for genetic investigations. It is easy to cultivate, and Mendel had a monastery garden and a greenhouse at his disposal. Peas grow relatively rapidly, completing an entire generation in a single growing season. Mendel started with 34 varieties of peas and spent two years selecting those varieties that he would use in his experiments $[20]$. In $[7,10]$, Davvaz et al. studied the connection between weak algebraic 
hyperstructures and inheritance.

The aim of this paper is to investigate a new connection between fuzzy sets and biological inheritance. More precisely, we consider the set of phenotypes of $F_{2}$ under mating and define a fuzzy subset of it. Our paper is constructed as follows: after an introduction, Section 2 presents some definitions that are used throughout the paper. Section 3 presents fuzzy sets and join spaces associated to the set of phenotypes of $F_{2}$ for the cases of simple and incomplete inheritance. Finally, Section 4 presents fuzzy sets and join spaces associated to the set of phenotypes of $F_{2}$ for some examples of non Mendelian inheritance.

Throughout this paper, parents is denoted by $P$, first generation by $F_{1}$ and second generation by $F_{2}$.

\section{Basic definitions}

In this section, we present some definitions related to hyperstructures (see [1]), fuzzy sets (see $[2,3,29]$ ) and to biological inheritance (see $[11,12,18]$ ) that are used throughout the paper.

Let $H$ be a non-empty set. Then, a mapping $\circ: H \times H \rightarrow \mathcal{P}^{*}(H)$ is called a binary hyperoperation on $H$, where $\mathcal{P}^{*}(H)$ is the family of all non-empty subsets of $H$. The couple $(H, \circ)$ is called a hypergroupoid. In the above definition, if $A$ and $B$ are two non-empty subsets of $H$ and $x \in H$, then we define:

$$
A \circ B=\bigcup_{a \in A, b \in B} a \circ b, x \circ A=\{x\} \circ A \text { and } A \circ x=A \circ\{x\} .
$$

An element $e \in H$ is called an identity of $(H, \circ)$ if $x \in x \circ e \cap e \circ x$, for all $x \in H$; it is called a scalar identity of $(H, \circ)$ if $x \circ e=e \circ x=\{x\}$, for all $x \in H$. If $e$ is a scalar identity of $(H, \circ)$, then $e$ is the unique identity of $(H, \circ)$. The hypergroupoid $(H, \circ)$ is said to be commutative if $x \circ y=y \circ x$, for all $x, y \in H$. A hypergroupoid $(H, \circ)$ is called a semihypergroup if for every $x, y, z \in H$, we have $x \circ(y \circ z)=(x \circ y) \circ z$ and is called a quasihypergroup if for every $x \in H, x \circ H=H=H \circ x$. This condition is called the reproduction axiom. The couple $(H, \circ)$ is called a hypergroup if it is a semihypergroup and a quasihypergroup. A canonical hypergroup [19] is a non-empty set $H$ endowed with a hyperoperation $\circ: H \times H \rightarrow \mathcal{P}^{*}(H)$, satisfying the following properties: (1) for any $x, y, z \in H, x \circ(y \circ z)=(x \circ y) \circ z$, (2) for any $x, y \in H, x \circ y=y \circ x,(3)$ there exists $\imath \in H$ such that $\imath \circ x=x \circ \imath=x$, for any $x \in H,(4)$ for every $x \in H$, there exists a unique element $x^{\prime}$ (or denote by $x^{-1}$ ) and we call it the inverse of $\left.x\right)$, (5) $z \in x \circ y$ implies that $y \in x^{\prime} \circ z$ and $x \in z \circ y^{\prime}$, that is $(H, \circ)$ is reversible. Two hypergroups $(H, \circ)$ and $(K, \star)$ are said to be isomorphic hypergroups if there exists a bijective function $f: H \rightarrow K$ such that $f(x \circ y)=f(x) \star f(y)$ for all $x, y \in H$.

Join spaces were introduced by W. Prenowitz [21, 22] and then applied by him and J. Jantosciak [23] in Euclidian as well as in non Euclidian geometry. Also, see $[15,16]$. Using this notion, several branches of non Euclidian geometry were rebuilt: descriptive geometry, projective geometry and spherical geometry. Then, 
several important examples of join spaces have been constructed in connection with binary relations, graphs, lattices, rough sets. In order to define a join space, we need the following notation: If $a, b$ are elements of a hypergroupoid $(H, \circ)$, then we denote $a / b=\{x \in H \mid a \in x \circ b\}$. A commutative hypergroup $(A, \star)$ is called a join space if for all $\alpha_{1}, \alpha_{2}, \alpha_{3}, \alpha_{4} \in A$ the following implication is true

$$
\alpha_{1} / \alpha_{2} \cap \alpha_{3} / \alpha_{4} \neq \varnothing \Rightarrow \alpha_{1} \star \alpha_{4} \cap \alpha_{2} \star \alpha_{3} \neq \varnothing .
$$

The first connection between fuzzy sets and hyperstructures was established by Corsini, when he defined a hyperoperation by means of fuzzy subsets. More precisely, let $\mu: H \rightarrow[0,1]$ be a fuzzy subset of a nonempty set $H$. Define on $H$ the hyperoperation $\star_{1}$, setting, for any $x, y \in H$,

$$
\left(w^{\prime}\right): x \star_{1} y=y \star_{1} x=\{z \in H: \min (\mu(x), \mu(y)) \leq \mu(z) \leq \max (\mu(x), \mu(y))\} .
$$

The associated hypergroupoid $\left({ }^{1} H, \star_{1}\right)$ is a join space. Also, he defined fuzzy subsets from hypergroups in the following manner: For any hypergroup $(H, \star)$, he defined a fuzzy subset $\mu: H \rightarrow[0,1]$ of $H$ in the following way: for $u \in H$ consider

$$
(w): \mu(u)=\frac{\sum_{(x, y) \in Q(u) \frac{1}{x \star y \mid}}}{q(u)},
$$

where $Q(u)=\left\{(a, b) \in H^{2}: u \in a \star b\right\}$ and $q(u)=|Q(u)|$ (see $\left.[2,3]\right)$.

Let $\left({ }^{1} H, \star_{1}\right)$ be the join space obtained by applying the fuzzy subset $\mu$ as defined in $\left(w^{\prime}\right)$. By using $(w)$ we get $\mu_{1}$ and using the same procedure as in $\left(w^{\prime}\right)$, from ${ }^{1} H$ we can obtain a membership function $\mu_{2}$ and the associated join space ${ }^{2} H$ and so on. A sequence of fuzzy sets and join spaces $\left(\left({ }^{i} H, \star_{i}\right), \mu_{i}\right)_{i \geq 1}$ is determined in this way. If two consecutive hypergroups of the obtained sequence are isomorphic, then the sequence stops. The length of the sequence of join spaces associated with $H$ is called the fuzzy grade of $H$. A hypergroupoid $H$ has a fuzzy grade $m \in \mathbb{N}$, written as $f . g(H)=m$ if for all $i, 0 \leq i<m$, the join spaces ${ }^{i} H$ and ${ }^{i+1} H$ are not isomorphic and for all $s>m,{ }^{s} H$ and ${ }^{m} H$ are isomorphic. If $f . g(H)=m$ and ${ }^{s} H={ }^{m} H$, for all $s>m$, we say that the strong fuzzy grade of $H$; s.f.g $(H)=m$. Such construction of join spaces is important for at least two reasons: it provides examples of hypergroup structures on a given set and it gives the possibility of studying fuzzy sets in an algebraic approach. On the other hand, the construction could start either from a fuzzy subset or from a hypergroup structure on a nonempty set $H$.

Inheritance involves the passing of discrete units of inheritance, or genes, from parents to offspring. Gregor Mendel [18], the first who introduced the notion of inheritance explicitly in 1865 , found that paired pea traits were either dominant or recessive. When pure bred parent plants $(P)$ were cross bred, dominant traits were always seen in the progeny, whereas recessive traits were hidden until the first generation $\left(F_{1}\right)$ hybrid plants were left to self pollinate. Mendel observed that in the second generation $\left(F_{2}\right)$, the traits of the $P$ generation reappeared. He concluded that traits were not blended but remained distinct in subsequent generations, which was contrary to scientific opinion at that time. Mendel didn't know about genes or 
discover genes, but he did speculate that there were two factors for each basic trait and that one factor was inherited from each parent. We now know that Mendel's inheritance factors are genes, or more specifically alleles (different variants of the same gene). In today's genetic language, a pure-breeding pea plant line is a homozygous (it has two identical copies of the same allele; $A A$ ) and an $F_{1}$ cross-bred pea plant is a heterozygous (it has two different alleles; $A B$ ). There are some exceptions to Mendel's principles, which have been discovered as our knowledge of genes and inheritance has increased. The principle of independent assortment doesn't apply if the genes are close together (or linked) on a chromosome. Also, alleles do not always interact in a standard dominant/recessive way (simple inheritance), particularly if they are codominant or have differences in expressivity or penetrance (incomplete inheritance). In the simple inheritance, we have two alleles ( $A$ dominant over $a$ ). The presence of the dominant allele in the genotype of an organism ( $A A$ or $A a$ ) leads to the presence of its corresponding phenotype and its absence $(a a)$ leads to the presence of the corresponding phenotype of the recessive trait. In the case of codominance, a cross between organisms with two different phenotypes (observed traits) produces offspring with a third phenotype that is a blending of the parental traits. For example, the cross of white and red flowers that results in the appearance of pink flowers (or white flowers with red spots) in the offspring is a good example on the codominance criteria.

Inheritance is linked to statistics in a way that we may find the probability of having a specific trait in the offspring. For example the monohybrid cross of parents with $A a$ genotypes in the case of simple inheritance gives offsprings having trait corresponding to $A$ with a probability $\frac{2}{3}$ and offsprings having trait corresponding to $a$ with a probability $\frac{1}{3}$.

\section{Fuzzy sets associated to simple and incomplete inheritance}

In this section, we consider hypothetical crosses of homozygous with independent number of alleles in the cases: simple inheritance, incomplete inheritance, simple and incomplete inheritance combined together. We define a fuzzy subset of the set of phenotypes of the second generation under mating $(x)$ and construct sequence of join spaces for each case.

Let $H$ be the set of phenotypes in $F_{2}$ and define $\mu: H \longrightarrow[0,1]$ by $\mu(x)=$ probability of $x$ for all $x \in H$. It is obvious that $\mu$ is a fuzzy subset of $H$.

\subsection{Simple inheritance}

Let $A_{i}$ be the dominant allele over $a_{i}$ for $i=1, \ldots, n$ and $\left\{A_{1}, \ldots, A_{n}\right\},\left\{a_{1}, \ldots, a_{n}\right\}$ be two sets of independent alleles. We consider first results for the Monohybrid cross

$(n=1)$ that differs in a single trait; a homozygous parent $\left(A_{1} A_{1}\right) \times$ a homozygous parent $\left(a_{1} a_{1}\right)$. The results of this hypothetical experiment can be summarized in the following way: 


$$
\begin{gathered}
\mathrm{P}: A_{1} A_{1} \times a_{1} a_{1} \\
F_{1}: A_{1} a_{1} \\
\text { and } \\
F_{1} \times F_{1}: A_{1} a_{1} \times A_{1} a_{1}
\end{gathered}
$$

$F_{2}: \widehat{B_{1}}$ (of genotype $A_{1} A_{1}$ or $A_{1} a_{1}$ ), $\widehat{B_{2}}$ (of genotype $a_{1} a_{1}$ ).

Let $H=\left\{\widehat{B_{1}}, \widehat{B_{2}}\right\}$ be the set of phenotypes in $F_{2}$. It is easy to see that $\mu\left(\widehat{B_{1}}\right)=\frac{2}{3}$ and $\mu\left(\widehat{B_{2}}\right)=\frac{1}{3}$.

Proposition 3.1. Let $H=\left\{\widehat{B_{1}}, \widehat{B_{2}}\right\}$ be the set of phenotypes in $F_{2}$. By definitions of $(w)$ and $\left(w^{\prime}\right)$, we have $\mu_{1}\left(\widehat{B_{1}}\right)=\mu_{1}\left(\widehat{B_{2}}\right)$ and S.F.G $(H)=2$.

Proof. Using $\left(w^{\prime}\right)$, we may present $\left({ }^{1} H, \star_{1}\right)$ by the following table:

\begin{tabular}{|c|c|c|}
\hline${ }^{1} H$ & $\widehat{B_{1}}$ & $\widehat{B_{2}}$ \\
\hline$\widehat{B_{1}}$ & $\left\{\widehat{B_{1}}\right\}$ & $H$ \\
\hline$\widehat{B_{2}}$ & & $\left\{\widehat{B_{2}}\right\}$ \\
\hline
\end{tabular}

Having $q\left(\widehat{B_{1}}\right)=q\left(\widehat{B_{2}}\right)=3$ and $A\left(\widehat{B_{1}}\right)=A\left(\widehat{B_{2}}\right)=\frac{1}{1}+\frac{2}{2}=2$ implies that $\mu_{1}\left(\widehat{B_{1}}\right)=\mu_{1}\left(\widehat{B_{2}}\right)=\frac{2}{3}$.

$\left({ }^{2} H, \star_{2}\right)$ can be presented by the following table:

\begin{tabular}{|c|c|c|}
\hline${ }^{2} H$ & $\widehat{B_{1}}$ & $\widehat{B_{2}}$ \\
\hline$\widehat{B_{1}}$ & $H$ & $H$ \\
\hline$\widehat{B_{2}}$ & & $H$ \\
\hline
\end{tabular}

It is clear that $\left({ }^{2} H, \star_{2}\right)$ is the total hypergroup. Therefore, S.F.G $(H)=2$.

We consider now results for the Dihybrid cross $(n=2)$ that differs in two traits; a homozygous parent $\left(A_{1} A_{1} A_{2} A_{2}\right) \times$ a homozygous parent $\left(a_{1} a_{1} a_{2} a_{2}\right)$. The results of this hypothetical experiment can be summarized in the following way:

$$
\begin{gathered}
\mathrm{P}: A_{1} A_{1} A_{2} A_{2} \times a_{1} a_{1} a_{2} a_{2} \\
F_{1}: A_{1} a_{1} A_{2} a_{2} \\
\text { and } \\
F_{1} \times F_{1}: A_{1} a_{1} A_{2} a_{2} \times A_{1} a_{1} A_{2} a_{2}
\end{gathered}
$$

$F_{2}: \widehat{B_{1}}$ (of genotype $A_{1} x_{1} A_{2} x_{2}$ ), $\widehat{B}_{2}$ (of genotype $A_{1} x_{1} a_{2} a_{2}$ ), $\widehat{B_{3}}$ (of genotype $a_{1} a_{1} A_{2} x_{2}$ ) and $\widehat{B_{4}}$ (of genotype $a_{1} a_{1} a_{2} a_{2}$ ).

Here, $x_{i} \in\left\{A_{i}, a_{i}\right\}$ for $i=1,2$.

Let $H=\left\{\widehat{B_{1}}, \widehat{B_{2}}, \widehat{B_{3}}, \widehat{B_{4}}\right\}$ be the set of phenotypes in $F_{2}$. It is easy to see that $\mu\left(\widehat{B_{1}}\right)=\frac{4}{9}, \mu\left(\widehat{B_{2}}\right)=\mu\left(\widehat{B_{3}}\right)=\frac{2}{9}$ and $\mu\left(\widehat{B_{4}}\right)=\frac{1}{9}$. 
Proposition 3.2. Let $H=\left\{\widehat{B_{1}}, \widehat{B_{2}}, \widehat{B_{3}}, \widehat{B_{4}}\right\}$. By definitions of $(w)$ and $\left(w^{\prime}\right)$, we have $\mu_{1}\left(\widehat{B_{2}}\right)=\mu_{1}\left(\widehat{B_{3}}\right)<\mu_{1}\left(\widehat{B_{1}}\right)=\mu_{1}\left(\widehat{B_{4}}\right)$.

Proof. Using $\left(w^{\prime}\right)$, we may present $\left({ }^{1} H, \star_{1}\right)$ by the following table:

\begin{tabular}{|c|c|c|c|c|}
\hline${ }^{1} H$ & $\widehat{B_{1}}$ & $\widehat{B_{2}}$ & $\widehat{B_{3}}$ & $\widehat{B_{4}}$ \\
\hline$\widehat{B_{1}}$ & $\left\{\widehat{B_{1}}\right\}$ & $\left\{\widehat{B_{1}}, \widehat{B_{2}}, \widehat{B_{3}}\right\}$ & $\left\{\widehat{B_{1}}, \widehat{B_{2}}, \widehat{B_{3}}\right\}$ & $H$ \\
\hline$\widehat{B_{2}}$ & & $\left\{\widehat{B_{2}}, \widehat{B_{3}}\right\}$ & $\left\{\widehat{B_{2}}, \widehat{B_{3}}\right\}$ & $\left\{\widehat{B_{2}}, \widehat{B_{3}}, \widehat{B_{4}}\right\}$ \\
\hline$\widehat{B_{3}}$ & & & $\left\{\widehat{B_{2}}, \widehat{B_{3}}\right\}$ & $\left\{\widehat{B_{2}}, \widehat{B_{3}}, \widehat{B_{4}}\right\}$ \\
\hline$\widehat{B_{4}}$ & & & & $\left\{\widehat{B_{4}}\right\}$ \\
\hline
\end{tabular}

Having $q\left(\widehat{B_{1}}\right)=q\left(\widehat{B_{4}}\right)=7, A\left(\widehat{B_{1}}\right)=A\left(\widehat{B_{4}}\right)=\frac{1}{1}+\frac{2}{3}+\frac{2}{3}+\frac{2}{4}=\frac{17}{6}, q\left(\widehat{B_{2}}\right)=$ $q\left(\widehat{B_{3}}\right)=14, A\left(\widehat{B_{2}}\right)=A\left(\widehat{B_{3}}\right)=\frac{2}{3}+\frac{2}{3}+\frac{2}{4}+\frac{1}{2}+\frac{2}{2}+\frac{1}{2}+\frac{2}{3}+\frac{2}{3}=\frac{31}{6}$ implies that $\mu_{1}\left(\widehat{B_{1}}\right)=\mu_{1}\left(\widehat{B_{4}}\right)=\frac{17}{42}$ and $\mu_{1}\left(\widehat{B_{2}}\right)=\mu_{1}\left(\widehat{B_{3}}\right)=\frac{31}{84}$.

Proposition 3.3. Let $H=\left\{\widehat{B_{1}}, \widehat{B_{2}}, \widehat{B_{3}}, \widehat{B_{4}}\right\}$. By definitions of $(w)$ and $\left(w^{\prime}\right)$, we have $\mu_{2}\left(\widehat{B_{1}}\right)=\mu_{2}\left(\widehat{B_{2}}\right)=\mu_{2}\left(\widehat{B_{3}}\right)=\mu_{2}\left(\widehat{B_{4}}\right)$ and S.F.G $(H)=3$.

Proof. We may present $\left({ }^{2} H, \star_{2}\right)$ by the following table:

\begin{tabular}{|c|c|c|c|c|}
\hline${ }^{2} H$ & $\widehat{B_{1}}$ & $\widehat{B_{2}}$ & $\widehat{B_{3}}$ & $\widehat{B_{4}}$ \\
\hline$\widehat{B_{1}}$ & $\left\{\widehat{B_{1}}, \widehat{B_{4}}\right\}$ & $H$ & $H$ & $\left\{\widehat{B_{1}}, \widehat{B_{4}}\right\}$ \\
\hline$\widehat{B_{2}}$ & & $\left\{\widehat{B_{2}}, \widehat{B_{3}}\right\}$ & $\left\{\widehat{B_{2}}, \widehat{B_{3}}\right\}$ & $H$ \\
\hline$\widehat{B_{3}}$ & & & $\left\{\widehat{B_{2}}, \widehat{B_{3}}\right\}$ & $H$ \\
\hline$\widehat{B_{4}}$ & & & & $\left\{\widehat{B_{1}}, \widehat{B_{4}}\right\}$ \\
\hline
\end{tabular}

Simple computations shows that $q\left(\widehat{B_{1}}\right)=g\left(\widehat{B_{2}}\right)=q\left(\widehat{B_{3}}\right)=q\left(\widehat{B_{4}}\right), A\left(\widehat{B_{1}}\right)=$ $A\left(\widehat{B_{2}}\right)=A\left(\widehat{B_{3}}\right)=A\left(\widehat{B_{4}}\right)$ and thus $\mu_{2}\left(\widehat{B_{1}}\right)=\mu_{2}\left(\widehat{B_{2}}\right)=\mu_{2}\left(\widehat{B_{3}}\right)=\mu_{2}\left(\widehat{B_{4}}\right)$. The latter implies that $\left({ }^{3} H, \star_{3}\right)$ is the total hypergroup and hence, S.F.G $(H)=3$.

\subsection{Case of incomplete inheritance}

Let $B_{i}$ and $\overline{B_{i}}$ be codominant alleles for $i=1, \ldots, n$ and $\left\{B_{1}, \ldots, B_{n}\right\},\left\{\overline{B_{1}}, \ldots, \overline{B_{n}}\right\}$ be two sets of independent alleles. We consider first results for the Monohybrid cross $(n=1)$ that differs in a single trait; a homozygous parent $\left(B_{1} B_{1}\right) \times$ a homozygous parent $\left(\overline{B_{1}} \overline{B_{1}}\right)$. The results of this hypothetical experiment can be summarized in the following way: 


$$
\begin{gathered}
\mathrm{P}: B_{1} B_{1} \times \overline{B_{1}} \overline{B_{1}} \\
F_{1}: B_{1} \overline{B_{1}} \\
\text { and } \\
F_{1} \times F_{1}: B_{1} \overline{B_{1}} \times B_{1} \overline{B_{1}}
\end{gathered}
$$

$F_{2}: \widehat{B_{1}}$ (of genotype $B_{1} B_{1}$ ), $\widehat{B_{2}}$ (of genotype $B_{1} \overline{B_{1}}$ ) and $\widehat{B_{3}}$ (of genotype $\overline{B_{1}} \overline{B_{1}}$ ).

Let $H=\left\{\widehat{B_{1}}, \widehat{B_{2}}, \widehat{B_{3}}\right\}$ be the set of phenotypes in $F_{2}$. It is easy to see that $\mu\left(\widehat{B_{1}}\right)=$ $\mu\left(\widehat{B_{2}}\right)=\mu\left(\widehat{B_{3}}\right)=\frac{1}{3}$.

Proposition 3.4. Let $H=\left\{\widehat{B_{1}}, \widehat{B_{2}}, \widehat{B_{3}}\right\}$. definitions of $(w)$ and $\left(w^{\prime}\right)$, we have $\mu_{1}\left(\widehat{B_{1}}\right)=\mu_{1}\left(\widehat{B_{2}}\right)=\mu_{1}\left(\widehat{B_{3}}\right)$ and S.F.G $(H)=1$.

Proof. Using $\left(w^{\prime}\right)$, we may present $\left({ }^{1} H, \star_{1}\right)$ by the following table:

\begin{tabular}{|c|c|c|c|}
\hline${ }^{1} H$ & $\widehat{B_{1}}$ & $\widehat{B_{2}}$ & $\widehat{B_{3}}$ \\
\hline$\widehat{B_{1}}$ & $H$ & $H$ & $H$ \\
\hline$\widehat{B_{2}}$ & & $H$ & $H$ \\
\hline$\widehat{B_{3}}$ & & & $H$ \\
\hline
\end{tabular}

It is clear that $\left({ }^{1} H, \star_{1}\right)$ is the total hypergroup and thus $S . F \cdot G(H)=1$.

We give next a generalization of Proposition 3.4 by considering the n- hybrid case of incomplete inheritance that differs in $n$ traits; a homozygous parent $\left(B_{1} B_{1} \ldots B_{n} B_{n}\right)$ $\times$ a homozygous parent $\left(\overline{B_{1}} \overline{B_{1}} \ldots \overline{B_{n}} \overline{B_{n}}\right)$. The results of this hypothetical experiment can be summarized in the following way:

$$
\begin{gathered}
\text { P: } B_{1} B_{1} \ldots B_{n} B_{n} \times \overline{B_{1}} \overline{B_{1}} \ldots \overline{B_{1}} \overline{B_{1}} \\
F_{1}: B_{1} \overline{B_{1}} \ldots B_{n} \overline{B_{n}} \\
F_{1} \times F_{1}: B_{1} \overline{B_{1}} \ldots \overline{B_{n}} \overline{B_{n}} \times B_{1} \overline{B_{1}} \ldots B_{n} \overline{B_{n}}
\end{gathered}
$$

$F_{2}: \widehat{B_{1}}$ (of genotype $B_{1} B_{1} \ldots B_{n} B_{n}$ ), $\widehat{B_{2}}$ (of genotype $B_{1} B_{1} \ldots B_{n-1} B_{n-1} B_{n} \overline{B_{n}}$ ), $\ldots$, and $\widehat{B_{k}}$ (of genotype $\overline{B_{1}} \overline{B_{1}} \ldots \overline{B_{n}} \overline{B_{n}}$ ).

The number of different phenotypes is $k=3^{n}$. Let $H=\left\{\widehat{B_{1}}, \ldots, \widehat{B_{k}}\right\}$ be the set of phenotypes in $F_{2}$. It is easy to see that $\mu\left(\widehat{B_{1}}\right)=\mu\left(\widehat{B_{2}}\right)=\ldots=\mu\left(\widehat{B_{k}}\right)=\frac{1}{k}$.

Theorem 3.1. Let $H=\left\{\widehat{B_{1}}, \ldots, \widehat{B_{k}}\right\}$. By definitions of $(w)$ and $\left(w^{\prime}\right)$, we have $\mu_{1}\left(\widehat{B_{1}}\right)=\ldots=\mu_{1}\left(\widehat{B_{k}}\right)$ and S.F.G $(H)=1$.

Proof. Using the definition of $\left(w^{\prime}\right)$, we have $\widehat{B_{i}} \star_{1} \widehat{B_{j}}=\left\{z \in H: \mu(z)=\frac{1}{k}\right\}=H$ for all $i, j \in\{1, \ldots, k\}$. Thus, $\left({ }^{1} H, \star_{1}\right)$ is the total hypergroup. 


\subsection{Case of simple and incomplete inheritance combined together}

Let $A_{i}$ be the dominant allele over $a_{i}$ for $i=1, \ldots, m$ and $B_{j}, \overline{B_{j}}$ be the codominant alleles for $j=1, \ldots, n$. This case can be given by considering the $(m+n)$ - hybrid case that differs in $(m+n)$ traits; a homozygous parent $\left(A_{1} A_{1} \ldots A_{m} A_{m} B_{1} B_{1} \ldots B_{n} B_{n}\right)$ $\times$ a homozygous parent $\left(a_{1} a_{1} \ldots a_{m} a_{m} \overline{B_{1}} \overline{B_{1}} \ldots \overline{B_{n}} \overline{B_{n}}\right)$.

We consider first the case $m=n=1$. The results of this hypothetical experiment can be summarized in the following way:

$$
\begin{gathered}
\mathrm{P}: A_{1} A_{1} B_{1} B_{1} \times a_{1} a_{1} \overline{B_{1}} \overline{B_{1}} \\
F_{1}: A_{1} a_{1} B_{1} \overline{B_{1}} \\
\text { and } \\
F_{1} \times F_{1}: A_{1} a_{1} B_{1} \overline{B_{1}} \times A_{1} a_{1} B_{1} \overline{B_{1}}
\end{gathered}
$$

$F_{2}: \widehat{B_{1}}$ (of genotype $A_{1} x_{1} B_{1} B_{1}$ ), $\widehat{B_{2}}$ (of genotype $A_{1} x_{1} B_{1} \overline{B_{1}}$ ), $\widehat{B_{3}}$ (of genotype $A_{1} x_{1} \overline{B_{1}} \overline{B_{1}}$ ), $\widehat{B_{4}}$ (of genotype $a_{1} a_{1} B_{1} B_{1}$ ), $\widehat{B_{5}}$ (of genotype $a_{1} a_{1} B_{1} \overline{B_{1}}$ ) and $\widehat{B_{6}}$ (of genotype $a_{1} a_{1} \overline{B_{1}} \overline{B_{1}}$ ).

Here. $x_{1} \in\left\{A_{1}, a_{1}\right\}$.

Let $H=\left\{\widehat{B_{1}}, \widehat{B_{2}}, \widehat{B_{3}}, \widehat{B_{4}}, \widehat{B_{5}}, \widehat{B_{6}}\right\}$ be the set of phenotypes in $F_{2}$. It is easy to see that $\mu\left(\widehat{B_{1}}\right)=\mu\left(\widehat{B_{3}}\right)=\mu\left(\widehat{B_{2}}\right)=\frac{2}{9}$ and $\mu\left(\widehat{B_{4}}\right)=\mu\left(\widehat{B_{5}}\right)=\mu\left(\widehat{B_{6}}\right)=\frac{1}{9}$.

Proposition 3.5. Let $H=\left\{\widehat{B_{1}}, \widehat{B_{2}}, \widehat{B_{3}}, \widehat{B_{4}}, \widehat{B_{5}}, \widehat{B_{6}}\right\}$. By definitions of $(w)$ and $\left(w^{\prime}\right)$, we have $\mu_{1}\left(\widehat{B_{1}}\right)=\mu_{1}\left(\widehat{B_{2}}\right)=\mu_{1}\left(\widehat{B_{3}}\right)=\mu_{1}\left(\widehat{B_{4}}\right)=\mu_{1}\left(\widehat{B_{5}}\right)=\mu_{1}\left(\widehat{B_{6}}\right)$ and S.F.G $(H)=2$.

Proof. The table below represents $\left({ }^{1} H, \star_{1}\right)$ :

\begin{tabular}{|c|c|c|c|c|c|c|}
\hline${ }^{1} H$ & $\widehat{B_{1}}$ & $\widehat{B_{2}}$ & $\widehat{B_{3}}$ & $\widehat{B_{4}}$ & $\widehat{B_{5}}$ & $\widehat{B_{6}}$ \\
\hline$\widehat{B_{1}}$ & $\left\{\widehat{B_{1}}, \widehat{B_{2}}, \widehat{B_{3}}\right\}$ & $\left\{\widehat{B_{1}}, \widehat{B_{2}}, \widehat{B_{3}}\right\}$ & $\left\{\widehat{B_{1}}, \widehat{B_{2}}, \widehat{B_{3}}\right\}$ & $H$ & $H$ & $H$ \\
\hline$\widehat{B_{2}}$ & & $\left\{\widehat{B_{1}}, \widehat{B_{2}}, \widehat{B_{3}}\right\}$ & $\left\{\widehat{B_{1}}, \widehat{B_{2}}, \widehat{B_{3}}\right\}$ & $H$ & $H$ & $H$ \\
\hline$\widehat{B_{3}}$ & & & $\left\{\widehat{B_{1}}, \widehat{B_{2}}, \widehat{B_{3}}\right\}$ & $H$ & $H$ & $H$ \\
\hline$\widehat{B_{4}}$ & & & & $\left\{\widehat{B_{4}}, \widehat{B_{5}}, \widehat{B_{6}}\right\}$ & $\left\{\widehat{B_{4}}, \widehat{B_{5}}, \widehat{B_{6}}\right\}$ & $\left\{\widehat{B_{4}}, \widehat{B_{5}}, \widehat{B_{6}}\right\}$ \\
\hline$\widehat{B_{5}}$ & & & & & $\left\{\widehat{B_{4}}, \widehat{B_{5}}, \widehat{B_{6}}\right\}$ & $\left\{\widehat{B_{4}}, \widehat{B_{5}}, \widehat{B_{6}}\right\}$ \\
\hline$\widehat{B_{6}}$ & & & & & & \\
\hline
\end{tabular}

It is easy to see that $\mu_{1}\left(\widehat{B_{1}}\right)=\mu_{1}\left(\widehat{B_{2}}\right)=\mu_{1}\left(\widehat{B_{3}}\right)=\mu_{1}\left(\widehat{B_{4}}\right)=\mu_{1}\left(\widehat{B_{5}}\right)=\mu_{1}\left(\widehat{B_{6}}\right)$ and that $\left({ }^{2} H, \star_{1}\right)$ is a total hypergroup. Therefore, S.F.G $(H)=2$.

We consider next the case $m=1$ and $n \geq 1$. The results of this hypothetical experiment can be summarized in the following way: 


$$
\begin{gathered}
\mathrm{P}: A_{1} A_{1} B_{1} B_{1} \ldots B_{n} B_{n} \times a_{1} a_{1} \overline{B_{1}} \overline{B_{1}} \ldots \overline{B_{n}} \overline{B_{n}} \\
F_{1}: A_{1} a_{1} B_{1} \overline{B_{1}} \ldots B_{n} \overline{B_{n}} \\
F_{1} \times F_{1}: A_{1} a_{1} B_{1} \overline{B_{1}} \ldots B_{n} \overline{B_{n}} \times A_{1} a_{1} B_{1} \overline{B_{1}} \ldots B_{n} \overline{B_{n}} .
\end{gathered}
$$

Let $H=\left\{\widehat{B_{1}}, \widehat{B_{2}}, \ldots, \widehat{B_{s}}\right\}$ be the set of phenotypes in $F_{2}$ where $s=2 k$ is the number of different phenotypes in $F_{2}, k=3^{n}, \widehat{B_{1}}, \ldots, \widehat{B_{k}}$ are phenotypes whose corresponding genotypes are given by $A_{1} x_{1} y_{1} y_{1}^{\prime} \ldots y_{n} y_{n}^{\prime}$ and $\widehat{B_{k+1}}, \ldots, \widehat{B_{2 k}}$ are phenotypes whose corresponding genotypes are given by $a_{1} a_{1} y_{1} y_{1}^{\prime} \ldots y_{n} y_{n}^{\prime}$. Where $x_{1} \in\left\{A_{1}, a_{1}\right\},\left\{y_{i}, y_{i}^{\prime}\right\} \subseteq\left\{B_{i}, \overline{B_{i}}\right\}$ for $i=1, \ldots, n$. It is easy to see that $\mu\left(\widehat{B_{1}}\right)=$ $\ldots=\mu\left(\widehat{B_{k}}\right)=\frac{2}{3^{n+1}}$ and $\mu\left(\widehat{B_{k+1}}\right)=\ldots=\mu\left(\widehat{B_{2 k}}\right)=\frac{1}{3^{n+1}}$.

Theorem 3.2. Let $H=\left\{\widehat{B_{1}}, \ldots, \widehat{B_{s}}\right\}$. By definition of $(w)$ and $\left(w^{\prime}\right)$, we have $\mu_{1}\left(\widehat{B_{1}}\right)=\mu_{1}\left(\widehat{B_{2}}\right)=\ldots=\mu_{1}\left(\widehat{B_{s}}\right)$ and S.F.G $(H)=2$.

Proof. Using $\left(w^{\prime}\right),\left({ }^{1} H, \star_{1}\right)$ may be constructed as follows:

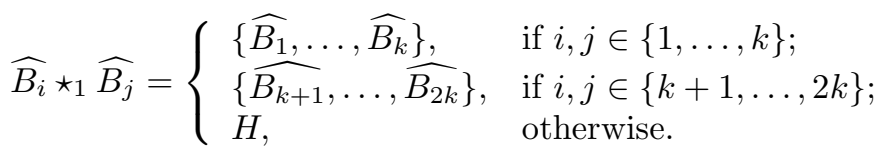

It is easy to see that $\left({ }^{2} H, \star_{2}\right)$ is the total hypergroup. Therefore, S.F.G $(H)=2$.

We consider next the case $m=2$ and $n=1$. The results of this hypothetical experiment can be summarized in the following way:

$$
\begin{gathered}
\text { P: } A_{1} A_{1} A_{2} A_{2} B_{1} B_{1} \times a_{1} a_{1} a_{2} a_{2} \overline{B_{1}} \overline{B_{1}} \\
F_{1}: A_{1} a_{1} A_{2} a_{2} B_{1} \overline{B_{1}} \\
\text { and } \\
F_{1} \times F_{1}: A_{1} a_{1} A_{2} a_{2} B_{1} \overline{B_{1}} \times A_{1} a_{1} A_{2} a_{2} B_{1} \overline{B_{1}} .
\end{gathered}
$$

Let $H=\left\{\widehat{B_{1}}, \widehat{B_{2}}, \ldots, \widehat{B_{12}}\right\}$ be the set of phenotypes in $F_{2}$ where $\widehat{B_{1}}, \widehat{B_{2}}, \widehat{B_{3}}$ are phenotypes whose corresponding genotypes are given by

$$
A_{1} x_{1} A_{2} x_{2} y_{1} y_{1}^{\prime}, \widehat{B_{4}}, \ldots, \widehat{B_{9}}
$$

are phenotypes whose corresponding genotypes are given by $A_{1} x_{1} a_{2} a_{2} y_{1} y_{1}^{\prime}$ or by $a_{1} a_{1} A_{2} x_{2} y_{1} y_{1}^{\prime}$ and $\widehat{B_{10}}, \widehat{B_{11}}, \widehat{B_{12}}$ are phenotypes whose corresponding genotypes are given by $a_{1} a_{1} a_{2} a_{2} y_{1} y_{1}^{\prime}$. Where $x_{i} \in\left\{A_{i}, a_{i}\right\}$ for $i=1,2$ and $\left\{y_{1}, y_{1}^{\prime}\right\} \subseteq\left\{B_{1}, \overline{B_{1}}\right\}$. It is easy to see that $\mu\left(\widehat{B_{1}}\right)=\mu\left(\widehat{B_{2}}\right)=\mu\left(\widehat{B_{3}}\right)=\frac{4}{27}, \mu\left(\widehat{B_{4}}\right)=\ldots=\mu\left(\widehat{B_{9}}\right)=\frac{2}{27}$ and $\mu\left(\widehat{B_{10}}\right)=\mu\left(\widehat{B_{11}}\right)=\mu\left(\widehat{B_{12}}\right)=\frac{1}{27}$.

Proposition 3.6. Let $H=\left\{\widehat{B_{1}}, \ldots, \widehat{B_{12}}\right\}$. By definition of $(w)$, we have $\mu_{1}\left(\widehat{B_{4}}\right)=\ldots=\mu_{1}\left(\widehat{B_{9}}\right)<\mu_{1}\left(\widehat{B_{1}}\right)=\mu_{1}\left(\widehat{B_{2}}\right)=\mu_{1}\left(\widehat{B_{3}}\right)=\mu_{1}\left(\widehat{B_{10}}\right)=\mu_{1}\left(\widehat{B_{11}}\right)=\mu_{1}\left(\widehat{B_{12}}\right)$. 
Proof. The table below represents $\left({ }^{1} H, \star_{1}\right)$ :

\begin{tabular}{|c|c|c|c|c|c|c|c|c|c|c|c|c|}
\hline${ }^{1} H$ & $\widehat{B_{1}}$ & $\widehat{B_{2}}$ & $\widehat{B_{3}}$ & $\widehat{B_{4}}$ & $\widehat{B_{5}}$ & $\widehat{B_{6}}$ & $\widehat{B_{7}}$ & $\widehat{B_{8}}$ & $\widehat{B_{9}}$ & $\widehat{B_{10}}$ & $\widehat{B_{11}}$ & $\widehat{B_{12}}$ \\
\hline$\widehat{B_{1}}$ & $M$ & $M$ & $M$ & $H \backslash R$ & $H \backslash R$ & $H \backslash R$ & $H \backslash R$ & $H \backslash R$ & $H \backslash R$ & $H$ & $H$ & $H$ \\
\hline$\widehat{B_{2}}$ & & $M$ & $M$ & $H \backslash R$ & $H \backslash R$ & $H \backslash R$ & $H \backslash R$ & $H \backslash R$ & $H \backslash R$ & $H$ & $H$ & $H$ \\
\hline$\widehat{B_{3}}$ & & & $M$ & $H \backslash R$ & $H \backslash R$ & $H \backslash R$ & $H \backslash R$ & $H \backslash R$ & $H \backslash R$ & $H$ & $H$ & $H$ \\
\hline$\widehat{B_{4}}$ & & & & $N$ & $N$ & $N$ & $N$ & $N$ & $N$ & $H \backslash M$ & $H \backslash M$ & $H \backslash M$ \\
\hline$\widehat{B_{5}}$ & & & & & $N$ & $N$ & $N$ & $N$ & $N$ & $H \backslash M$ & $H \backslash M$ & $H \backslash M$ \\
\hline$\widehat{B_{6}}$ & & & & & & $N$ & $N$ & $N$ & $N$ & $H \backslash M$ & $H \backslash M$ & $H \backslash M$ \\
\hline$\widehat{B_{7}}$ & & & & & & & $N$ & $N$ & $N$ & $H \backslash M$ & $H \backslash M$ & $H \backslash M$ \\
\hline$\widehat{B_{8}}$ & & & & & & & & $N$ & $N$ & $H \backslash M$ & $H \backslash M$ & $H \backslash M$ \\
\hline$\widehat{B_{9}}$ & & & & & & & & & $N$ & $H \backslash M$ & $H \backslash M$ & $H \backslash M$ \\
\hline$\widehat{B_{10}}$ & & & & & & & & & & $R$ & $R$ & $R$ \\
\hline$\widehat{B_{11}}$ & & & & & & & & & & & $R$ & $R$ \\
\hline$\widehat{B_{12}}$ & & & & & & & & & & & & $R$ \\
\hline
\end{tabular}

where $M=\left\{\widehat{B_{1}}, \widehat{B_{2}}, \widehat{B_{3}}\right\}, R=\left\{\widehat{B_{10}}, \widehat{B_{11}}, \widehat{B_{12}}\right\}$ and $N=H \backslash(M \cup R)$.

We have that $q\left(\widehat{B_{1}}\right)=63, A\left(\widehat{B_{1}}\right)=\frac{9}{3}+\frac{18}{12}+\frac{36}{9}, q\left(\widehat{B_{4}}\right)=126$ and $A\left(\widehat{B_{4}}\right)=$ $\frac{36}{6}+\frac{72}{9}+\frac{18}{12}$. Simple calculations implies that

$$
\mu_{1}\left(\widehat{B_{1}}\right)=\mu_{1}\left(\widehat{B_{2}}\right)=\mu_{1}\left(\widehat{B_{3}}\right)=\mu_{1}\left(\widehat{B_{10}}\right)=\mu_{1}\left(\widehat{B_{11}}\right)=\mu_{1}\left(\widehat{B_{12}}\right)=\frac{17}{126}
$$

and

$$
\mu_{1}\left(\widehat{B_{4}}\right)=\mu_{1}\left(\widehat{B_{5}}\right)=\mu_{1}\left(\widehat{B_{6}}\right)=\mu_{1}\left(\widehat{B_{7}}\right)=\mu_{1}\left(\widehat{B_{8}}\right)=\mu_{1}\left(\widehat{B_{9}}\right)=\frac{31}{252} .
$$

Proposition 3.7. Let $H=\left\{\widehat{B_{1}}, \ldots, \widehat{B_{12}}\right\}$. By definition of $(w)$, we have $\mu_{2}\left(\widehat{B_{1}}\right)=$ $\ldots=\mu_{2}\left(\widehat{B_{12}}\right)$ and S.F.G $(H)=3$.

Proof. We may present $\left({ }^{2} H, \star_{2}\right)$ by the following table: 


\begin{tabular}{|c|c|c|c|c|c|c|c|c|c|c|c|c|}
\hline${ }^{2} H$ & $\widehat{B_{1}}$ & $\widehat{B_{2}}$ & $\widehat{B_{3}}$ & $\widehat{B_{4}}$ & $\widehat{B_{5}}$ & $\widehat{B_{6}}$ & $\widehat{B_{7}}$ & $\widehat{B_{8}}$ & $\widehat{B_{9}}$ & $\widehat{B_{10}}$ & $\widehat{B_{11}}$ & $\widehat{B_{12}}$ \\
\hline$\widehat{B_{1}}$ & $P$ & $P$ & $P$ & $H$ & $H$ & $H$ & $H$ & $H$ & $H$ & $P$ & $P$ & $P$ \\
\hline$\widehat{B_{2}}$ & & $P$ & $P$ & $H$ & $H$ & $H$ & $H$ & $H$ & $H$ & $P$ & $P$ & $P$ \\
\hline$\widehat{B_{3}}$ & & & $P$ & $H$ & $H$ & $H$ & $H$ & $H$ & $H$ & $P$ & $P$ & $P$ \\
\hline$\widehat{B_{4}}$ & & & & $Q$ & $Q$ & $Q$ & $Q$ & $Q$ & $Q$ & $H$ & $H$ & $H$ \\
\hline$\widehat{B_{5}}$ & & & & & $Q$ & $Q$ & $Q$ & $Q$ & $Q$ & $H$ & $H$ & $H$ \\
\hline$\widehat{B_{6}}$ & & & & & & $Q$ & $Q$ & $Q$ & $Q$ & $H$ & $H$ & $H$ \\
\hline$\widehat{B_{7}}$ & & & & & & & $Q$ & $Q$ & $Q$ & $H$ & $H$ & $H$ \\
\hline$\widehat{B_{8}}$ & & & & & & & & $Q$ & $Q$ & $H$ & $H$ & $H$ \\
\hline$\widehat{B_{9}}$ & & & & & & & & & $Q$ & $H$ & $H$ & $H$ \\
\hline$\widehat{B_{10}}$ & & & & & & & & & & $P$ & $P$ & $P$ \\
\hline$\widehat{B_{11}}$ & & & & & & & & & & & $P$ & $P$ \\
\hline$\widehat{B_{12}}$ & & & & & & & & & & & & $P$ \\
\hline
\end{tabular}

where $P=\left\{\widehat{B_{1}}, \widehat{B_{2}}, \widehat{B_{3}}, \widehat{B_{10}}, \widehat{B_{11}}, \widehat{B_{12}}\right\}$ and $Q=\left\{\widehat{B_{4}}, \widehat{B_{5}}, \widehat{B_{6}}, \widehat{B_{7}}, \widehat{B_{8}}, \widehat{B_{9}}\right\}$. Simple computations implies that $\mu_{2}\left(\widehat{B_{1}}\right)=\ldots=\mu_{2}\left(\widehat{B_{12}}\right)=\frac{1}{9}$. Thus, $\left({ }^{3} H, \star_{3}\right)$ is the total hypergroup and hence S.F.G $(H)=3$.

We consider next the case $m=2$ and $n \geq 1$. The results of this hypothetical experiment can be summarized in the following way:

$$
\begin{gathered}
\mathrm{P}: A_{1} A_{1} A_{2} A_{2} B_{1} B_{1} \ldots B_{n} B_{n} \times a_{1} a_{1} a_{2} a_{2} \overline{B_{1}} \overline{B_{1}} \ldots \overline{B_{n}} \overline{B_{n}} \\
F_{1}: A_{1} a_{1} A_{2} a_{2} B_{1} \overline{B_{1}} \ldots B_{n} \overline{B_{n}} \\
F_{1} \times F_{1}: A_{1} a_{1} A_{2} a_{2} B_{1} \overline{B_{1}} \ldots B_{n} \overline{B_{n}} \times A_{1} a_{1} A_{2} a_{2} B_{1} \overline{B_{1}} \ldots B_{n} \overline{B_{n}} .
\end{gathered}
$$

Let $H=\left\{\widehat{B_{1}}, \widehat{B_{2}}, \ldots, \widehat{B_{r}}\right\}$ be the set of phenotypes in $F_{2}$ where $r=4 k, k=3^{n}$, $\widehat{B_{1}}, \ldots, \widehat{B_{k}}$ are phenotypes whose corresponding genotypes are given by

$$
A_{1} x_{1} A_{2} x_{2} y_{1} y_{1}^{\prime} \ldots y_{n} y_{n}^{\prime}, \widehat{B_{k+1}}, \ldots, \widehat{B_{3 k}}
$$

are phenotypes whose corresponding genotypes are given by $A_{1} x_{1} a_{2} a_{2} y_{1} y_{1}^{\prime} \ldots y_{n} y_{n}^{\prime}$ or by $a_{1} a_{1} A_{2} x_{2} y_{1} y_{1}^{\prime} \ldots y_{n} y_{n}^{\prime}$ and $\widehat{B_{3 k+1}}, \ldots, \widehat{B_{4 k}}$ are phenotypes whose corresponding genotypes are given by $a_{1} a_{1} a_{2} a_{2} y_{1} y_{1}^{\prime} \ldots y_{n} y_{n}^{\prime}$. Where $x_{i} \in\left\{A_{i}, a_{i}\right\}$ for $i=1,2$ and $\left\{y_{j}, y_{j}^{\prime}\right\} \subseteq\left\{B_{j}, \overline{B_{j}}\right\}$ for $j=1, \ldots, n$. It is easy to see that $\mu\left(\widehat{B_{1}}\right)=\ldots=$ $\mu\left(\widehat{B_{k}}\right)=\frac{4}{3^{n+2}}, \mu\left(\widehat{B_{k+1}}\right)=\ldots=\mu\left(\widehat{B_{3 k}}\right)=\frac{2}{3^{n+2}}$ and $\mu\left(\widehat{B_{3 k+1}}\right)=\ldots=\mu\left(\widehat{B_{4 k}}\right)=$ $\frac{1}{3^{n+2}}$. 
Proposition 3.8. Let $H=\left\{\widehat{B_{1}}, \ldots, \widehat{B_{s}}\right\}$ be the set of phenotypes of $F_{2}$. By definitions of $(w)$ and $\left(w^{\prime}\right)$, we have

$\mu_{1}\left(\widehat{B_{k+1}}\right)=\ldots=\mu_{1}\left(\widehat{B_{3 k}}\right)<\mu_{1}\left(\widehat{B_{1}}\right)=\ldots=\mu_{1}\left(\widehat{B_{k}}\right)=\mu_{1}\left(\widehat{B_{3 k+1}}\right)=\ldots=\mu_{1}\left(\widehat{B_{4 k}}\right)$.

Proof. The table below represents $\left({ }^{1} H, \star_{1}\right)$ :

\begin{tabular}{|c|c|c|c|c|c|c|c|c|c|}
\hline${ }^{1} H$ & $\widehat{B_{1}}$ & $\ldots$ & $\widehat{B_{k}}$ & $\widehat{B_{k+1}}$ & $\ldots$ & $\widehat{B_{3 k}}$ & $\widehat{B_{3 k+1}}$ & $\ldots$ & $\widehat{B_{4 k}}$ \\
\hline$\widehat{B_{1}}$ & $M_{1}$ & $\ldots$ & $M_{1}$ & $M_{1} \cup M_{2}$ & $\ldots$ & $M_{1} \cup M_{2}$ & $H$ & $\ldots$ & $H$ \\
\hline$\vdots$ & & $\ddots$ & $\vdots$ & $\vdots$ & $\vdots$ & $\vdots$ & $\vdots$ & $\vdots$ & $\vdots$ \\
\hline$\widehat{B_{k}}$ & & & $M_{1}$ & $M_{1} \cup M_{2}$ & $\ldots$ & $M_{1} \cup M_{2}$ & $H$ & $\ldots$ & $H$ \\
\hline$\widehat{B_{k+1}}$ & & & & $M_{2}$ & $\ldots$ & $M_{2}$ & $M_{2} \cup M_{3}$ & $\ldots$ & $M_{2} \cup M_{3}$ \\
\hline$\vdots$ & & & & & $\ddots$ & $\vdots$ & $\vdots$ & $\vdots$ & $\vdots$ \\
\hline$\widehat{B_{3 k}}$ & & & & & & $M_{2}$ & $M_{2} \cup M_{3}$ & $\ldots$ & $M_{2} \cup M_{3}$ \\
\hline$\widehat{B_{3 k+1}}$ & & & & & & & $M_{3}$ & $\ldots$ & $M_{3}$ \\
\hline$\vdots$ & & & & & & & & $\ddots$ & $\vdots$ \\
\hline$\widehat{B_{4 k}}$ & & & & & & & & & $M_{3}$ \\
\hline
\end{tabular}

where $M_{1}=\left\{\widehat{B_{1}}, \ldots, \widehat{B_{k}}\right\}, M_{2}=\left\{\widehat{B_{k+1}}, \ldots, \widehat{B_{3 k}}\right\}$ and $M_{3}=\left\{\widehat{B_{3 k+1}}, \ldots, \widehat{B_{4 k}}\right\}$.

It is easy to see that

$$
\mu_{1}\left(\widehat{B_{1}}\right)=\ldots=\mu_{1}\left(\widehat{B_{k}}\right)=\mu_{1}\left(\widehat{B_{3 k+1}}\right)=\ldots=\mu_{1}\left(\widehat{B_{4 k}}\right)
$$

and

$$
\mu_{1}\left(\widehat{B_{k+1}}\right)=\ldots=\mu_{1}\left(\widehat{B_{4 k}}\right) .
$$

We have that $q\left(\widehat{B_{1}}\right)=7 k^{2}, q\left(\widehat{B_{k+1}}\right)=14 k^{2}$. Simple computations shows that $A\left(\widehat{B_{1}}\right)=\frac{k^{2}}{\left|M_{1}\right|}+\frac{4 k^{2}}{\left|M_{1}\right|+\left|M_{2}\right|}+\frac{2 k^{2}}{|H|}=\frac{17 k}{6}$ and $A\left(\widehat{B_{k+1}}\right)=\frac{4 k^{2}}{\left|M_{2}\right|}+\frac{4 k^{2}}{\left|M_{1}\right|+\left|M_{2}\right|}+\frac{2 k^{2}}{|H|}+$ $\frac{4 k^{2}}{\left|M_{2}\right|+\left|M_{3}\right|}=\frac{31 k}{6}$. We get now

$$
\mu_{1}\left(\widehat{B_{1}}\right)=\ldots=\mu_{1}\left(\widehat{B_{k}}\right)=\mu_{1}\left(\widehat{B_{3 k+1}}\right)=\ldots=\mu_{1}\left(\widehat{B_{4 k}}\right)=\frac{17}{6 k}
$$

and

$$
\mu_{1}\left(\widehat{B_{k+1}}\right)=\ldots=\mu_{1}\left(\widehat{B_{3 k}}\right)=\frac{31}{84 k}
$$

Proposition 3.9. Let $H=\left\{\widehat{B_{1}}, \ldots, \widehat{B_{r}}\right\}$. By definition of $(w)$, we have $\mu_{2}\left(\widehat{B_{1}}\right)=$ $\ldots=\mu_{2}\left(\widehat{B_{r}}\right)$ and S.F.G $(H)=3$. 
Proof. We may present $\left({ }^{2} H, \star_{2}\right)$ by the following table:

\begin{tabular}{|c|c|c|c|c|c|c|c|c|c|}
\hline${ }^{2} H$ & $\widehat{B_{1}}$ & $\ldots$ & $\widehat{B_{k}}$ & $\widehat{B_{k+1}}$ & $\ldots$ & $\widehat{B_{3 k}}$ & $\widehat{B_{3 k+1}}$ & $\ldots$ & $\widehat{B_{4 k}}$ \\
\hline$\widehat{B_{1}}$ & $M_{1} \cup M_{3}$ & $\ldots$ & $M_{1} \cup M_{3}$ & $H$ & $\ldots$ & $H$ & $M_{1} \cup M_{3}$ & $\ldots$ & $M_{1} \cup M_{3}$ \\
\hline$\vdots$ & & $\ddots$ & $\vdots$ & $\vdots$ & $\vdots$ & $\vdots$ & $\vdots$ & $\vdots$ & $\vdots$ \\
\hline$\widehat{B_{k}}$ & & & $M_{1} \cup M_{3}$ & $H$ & $\ldots$ & $H$ & $M_{1} \cup M_{3}$ & $\ldots$ & $M_{1} \cup M_{3}$ \\
\hline$\widehat{B_{k+1}}$ & & & & $M_{2}$ & $\ldots$ & $M_{2}$ & $H$ & $\ldots$ & $H$ \\
\hline$\vdots$ & & & & & $\ddots$ & $\vdots$ & $\vdots$ & $\vdots$ & $\vdots$ \\
\hline$\widehat{B_{3 k}}$ & & & & & & $M_{2}$ & $H$ & $\ldots$ & $H$ \\
\hline$\widehat{B_{3 k+1}}$ & & & & & & & $M_{1} \cup M_{3}$ & $\ldots$ & $M_{1} \cup M_{3}$ \\
\hline$\vdots$ & & & & & & & & $\ddots$ & $\vdots$ \\
\hline$\widehat{B_{4 k}}$ & & & & & & & & & $M_{1} \cup M_{3}$ \\
\hline
\end{tabular}

where $M_{1}=\left\{\widehat{B_{1}}, \ldots, \widehat{B_{k}}\right\}, M_{2}=\left\{\widehat{B_{k+1}}, \ldots, \widehat{B_{3 k}}\right\}$ and $M_{3}=\left\{\widehat{B_{3 k+1}}, \ldots, \widehat{B_{4 k}}\right\}$. We have:

$$
q\left(\widehat{B_{i}}\right)=12 k^{2} \text { for } i=1, \ldots, r,
$$

and

$$
A(x)= \begin{cases}\frac{4 k^{2}}{\left|M_{1}\right|+\left|M_{3}\right|}+\frac{8 k^{2}}{|H|}=4 k, & \text { for } x \in M_{1} \cup M_{3} ; \\ \frac{4 k^{2}}{\left|M_{2}\right|}+\frac{8 k^{2}}{|H|}=4 k, & \text { for } x \in M_{2} .\end{cases}
$$

We get now that $A(x)=4 k$ for all $x \in H$. The latter implies that $\mu(x)=\frac{1}{3 k}$ for all $x \in H$. Therefore, $\left({ }^{3} H, \star_{3}\right)$ is the total hypergroup and S.F.G $G(H)=3$.

\section{Fuzzy sets associated to other types of inheritance}

In this section, we study some examples of different types of non- Mendelian inheritance (Epistasis, Supplementary gene and Inhibitory gene), define fuzzy subsets of them and construct sequence of join spaces for each type.

The fuzzy subset $\mu$ of the set of phenotypes in $F_{2}$ of each type is defined by $\mu(x)=$ probability of $x$ for all $x \in F_{2}$.

Example 4.1. Epistasis of dominant gene in the coat color of dogs. There are two allelomorphic pairs which may be named $A a$ and $B b, A$ and $B$ are dominant over $a$ and $b$ respectively. They interact as follows: $A x B y$ and $A x b b$ have phenotype white, $a a B y$ has phenotype black and $a a b b$ has phenotype brown. Here $x=A$ or $a$ and $y=B$ or $b$. The results of this experiment can be summarized in the following way:

$$
\begin{aligned}
& \text { P: } A A B B \otimes a a b b \\
& F_{1}: A a B b
\end{aligned}
$$




$$
\begin{gathered}
\text { and } \\
F_{1} \otimes F_{1}: A a B b \otimes A a B b \\
F_{2}: \text { White, Black, Brown. }
\end{gathered}
$$

White is denoted by $A_{1}$, Black by $A_{2}$ and Brown by $A_{3}$.

Let $H=\left\{A_{1}, A_{2}, A_{3}\right\}$ be the set of phenotypes in $F_{2}$. It is easy to see that $\mu\left(A_{1}\right)=$ $\frac{6}{9}, \mu\left(A_{2}\right)=\frac{2}{9}$ and $\mu\left(A_{3}\right)=\frac{1}{9}$.

Proposition 4.1. Let $H=\left\{A_{1}, A_{2}, A_{3}\right\}$. By definitions of $(w)$ and $\left(w^{\prime}\right)$, we have $\mu_{1}\left(A_{2}\right)<\mu_{1}\left(A_{1}\right)=\mu_{1}\left(A_{3}\right)$.

Proof. The table below represents $\left({ }^{1} H, \star_{1}\right)$ :

\begin{tabular}{|c|c|c|c|}
\hline${ }^{1} H$ & $A_{1}$ & $A_{2}$ & $A_{3}$ \\
\hline$A_{1}$ & $\left\{A_{1}\right\}$ & $\left\{A_{1}, A_{2}\right\}$ & $H$ \\
\hline$A_{2}$ & & $\left\{A_{2}\right\}$ & $\left\{A_{2}, A_{3}\right\}$ \\
\hline$A_{3}$ & & & $\left\{A_{3}\right\}$ \\
\hline
\end{tabular}

We have $q\left(A_{1}\right)=q\left(A_{3}\right)=5, q\left(A_{2}\right)=7, A\left(A_{1}\right)=A\left(A_{3}\right)=\frac{1}{1}+\frac{2}{2}+\frac{2}{3}=\frac{8}{3}$ and $A\left(A_{2}\right)=\frac{1}{1}+\frac{2}{2}+\frac{2}{2}+\frac{2}{3}=\frac{11}{3}$. Thus, $\mu_{1}\left(A_{1}\right)=\mu_{1}\left(A_{3}\right)=\frac{8}{15}$ and $\mu_{1}\left(A_{2}\right)=\frac{11}{21}$.

Proposition 4.2. Let $H=\left\{A_{1}, A_{2}, A_{3}\right\}$. By definition of $(w)$, we have $\mu_{2}\left(A_{1}\right)=$ $\mu_{2}\left(A_{3}\right)<\mu_{2}\left(A_{2}\right)$.

Proof. The table below represents $\left({ }^{2} H, \star_{1}\right)$ :

\begin{tabular}{|c|c|c|c|}
\hline${ }^{2} H$ & $A_{1}$ & $A_{2}$ & $A_{3}$ \\
\hline$A_{1}$ & $\left\{A_{1}, A_{3}\right\}$ & $H$ & $\left\{A_{1}, A_{3}\right\}$ \\
\hline$A_{2}$ & & $\left\{A_{2}\right\}$ & $H$ \\
\hline$A_{3}$ & & & $\left\{A_{1}, A_{3}\right\}$ \\
\hline
\end{tabular}

We have $q\left(A_{1}\right)=q\left(A_{3}\right)=8, q\left(A_{2}\right)=5, A\left(A_{1}\right)=A\left(A_{3}\right)=\frac{4}{2}+\frac{4}{3}=\frac{10}{3}$ and $A\left(A_{2}\right)=\frac{1}{1}+\frac{4}{3}=\frac{7}{3}$. Thus, $\mu_{2}\left(A_{1}\right)=\mu_{2}\left(A_{3}\right)=\frac{5}{12}$ and $\mu_{2}\left(A_{2}\right)=\frac{7}{15}$.

Proposition 4.3. Let $H=\left\{A_{1}, A_{2}, A_{3}\right\}$ be the set of phenotypes in $F_{2}$. Then S.F. $G(H)=2$.

Proof. The table below represents $\left({ }^{3} H, \star_{1}\right)$ : 


\begin{tabular}{|c|c|c|c|}
\hline${ }^{3} H$ & $A_{1}$ & $A_{2}$ & $A_{3}$ \\
\hline$A_{1}$ & $\left\{A_{1}, A_{3}\right\}$ & $H$ & $\left\{A_{1}, A_{3}\right\}$ \\
\hline$A_{2}$ & & $\left\{A_{2}\right\}$ & $H$ \\
\hline$A_{3}$ & & & $\left\{A_{1}, A_{3}\right\}$ \\
\hline
\end{tabular}

Having $\left({ }^{3} H, \star_{1}\right)=\left({ }^{2} H, \star_{1}\right)$ implies that S.F.G $(H)=2$.

Example 4.2. Supplementary gene, The anthocyanin pigmentation of flowers. The redtype anthocyanin color of many flowers is caused by two alleles which may be termed as $A a$ and $B b$. In the snapdragon (Antirrhinum) flower:

$A x B y$ is the genotype of magenta flower, $A x b b$ is the genotype of ivory flower and $a a B y, a a b b$ are the genotypes of white flower where $x=A$ or $a$ and $y=B$ or $b$. The results of this experiment can be summarized in the following way:

$$
\begin{gathered}
\mathrm{P}: A A B B \otimes a a b b \\
F_{1}: A a B b \\
\text { and } \\
F_{1} \otimes F_{1}: A a B b \otimes A a B b \\
F_{2}: \text { Magneta, Ivory, White. }
\end{gathered}
$$

Magneta is denoted by $B_{1}$, White by $B_{2}$ and Ivory by $B_{3}$.

Let $K=\left\{B_{1}, B_{2}, B_{3}\right\}$ be the set of phenotypes in $F_{2}$. It is easy to see that $\mu\left(B_{1}\right)=$ $\frac{4}{9}, \mu\left(B_{2}\right)=\frac{3}{9}$ and $\mu\left(B_{3}\right)=\frac{2}{9}$.

Theorem 4.1. Let $K=\left\{B_{1}, B_{2}, B_{3}\right\}$ be the set of phenotypes in $F_{2}$. Then S.F.G $(K)=2$.

Proof. Since $\mu\left(B_{3}\right)<\mu\left(B_{2}\right)<\mu\left(B_{1}\right)$ then using $\left(w^{\prime}\right)$, we may present $\left({ }^{1} K, \star_{1}\right)$ as follows:

\begin{tabular}{|c|c|c|c|}
\hline${ }^{1} K$ & $B_{1}$ & $B_{2}$ & $B_{3}$ \\
\hline$B_{1}$ & $\left\{B_{1}\right\}$ & $\left\{B_{1}, B_{2}\right\}$ & $H$ \\
\hline$B_{2}$ & & $\left\{B_{2}\right\}$ & $\left\{B_{2}, B_{3}\right\}$ \\
\hline$B_{3}$ & & & $\left\{B_{3}\right\}$ \\
\hline
\end{tabular}

It is easy to see that $\left({ }^{1} H, \star_{1}\right)$ (of Proposition 4.1, Example 4.1) and $\left({ }^{1} K, \star_{1}\right)$ are isomorphic. This implies that S.F.G $(K)=S . F . G(H)$. Therefore, S.F.G $(K)=2$ by Proposition 4.3.

Example 4.3. Inhibitory gene, Rice leaf. In some rice variety the presence of the gene $P$ causes its leaves to be colored deep purple. But if a gene $I$ is present then the purple 
color is inhibited and the leaf becomes normal green. The $I$ gene may be considered as epistatic over $P$. They interact as follows:

The genotypes IxPy, Ixpp, iipp correspond to green and the genotype iiPy corresponds to purple where $x=I$ or $i$ and $y=P$ or $p$. The results of this experiment can be summarized in the following way:

$$
\begin{gathered}
\text { P: } I I P P \otimes i i p p \\
F_{1}: I i P p \\
\text { and } \\
F_{1} \otimes F_{1}: I i P p \otimes I i P p \\
F_{2}: \text { Green, Purple. }
\end{gathered}
$$

Green is denoted by $G$ and Purple by $P$.

Let $L=\{G, P\}$ be the set of phenotypes in $F_{2}$. It is easy to see that $\mu(G)=\frac{7}{9}$ and $\mu(P)=\frac{2}{9}$.

Proposition 4.4. Let $L=\{G, P\}$ be the set of phenotypes in $F_{2}$. Then S.F.G $(L)=$ 2 .

Proof. The table below represents $\left({ }^{1} L, \star_{1}\right)$ :

\begin{tabular}{|c|c|c|}
\hline${ }^{1} L$ & $G$ & $P$ \\
\hline$G$ & $\{G\}$ & $H$ \\
\hline$P$ & & $\{P\}$ \\
\hline
\end{tabular}

It is easy to see that $\left({ }^{1} H, \star_{1}\right)$ (of Proposition 3.1) and $\left({ }^{1} L, \star_{1}\right)$ are isomorphic. Therefore S.F.G $(L)=$ S.F.G $(H)=2$.

\section{Conclusion}

After the introduction of hyperstructures and fuzzy sets by Marty and Zadeh there have been many researches that study their importance in different fields where one of these fields is biological inheritance. This paper studied a new relationship between hyperstructures, fuzzy sets and the phenotypes of the second generation $F_{2}$. Fuzzy subsets of $F_{2}$ were defined and join spaces associated to $F_{2}$ were constructed.

\section{REFEREN C ES}

1. P. Corsini, Prolegomena of Hypergroup Theory (Aviani Editor, 1993).

2. P. Corsini, Join spaces, power sets, fuzzy sets, Proceedings of the FifthInt. Congress of Algebraic Hyperstructures and Appl., 1993, Iasi, Romania, Hadronic Press, 1994. 
3. P. Corsini, A new connection between Hypergroups and Fuzzy Sets, Southeast Asian Bulletin of Mathematics, 27 (2003), 221-229.

4. P. Corsini and V. Leoreanu, Applications of hyperstructures theory, Advanced in Mathematics, Kluwer Academic Publisher, 2003.

5. C. Darwin, The Variation of Animals and Plants Under Domestication(1st American edition), New York: Orange Judd and Co. (1868).

6. B. Davvaz, Polygroup Theory and Related Systems, World Scientific Publishing Co. Pte. Ltd., Hackensack, NJ, 2013. viii+200 pp.

7. B. Davvaz, A. Dehghan Nezhad and M. Heidari, Inheritance examples of algebraic hyperstructures, Information Sciences, 224 (2013), 180-187.

8. B. Davvaz, V. Leoreanu-Fotea, Hyperring Theory and Applications, International Academic Press, USA, 2007.

9. A. Dehghan Nezhad, S.M. Moosavi Nejad, M. Nadjafikhah and B. Davvaz, A physical example of algebraic hyperstructures: Leptons, Indian Journal of Physics, 86(11) (2012), 1027-1032.

10. M. Ghadiri, B. Davvaz and R. Nekouian, $H_{v}$-Semigroup structure on $F_{2}$-offspring of a gene pool, International Journal of Biomathematics, 5(4) (2012) 1250011 (13 pages).

11. A. J. F. Griffith et al., it An Introduction to Genetic Analysis, 7th edn. (WH Freeman, New York, 2000).

12. L. H. Hartwell, L. Hood, M. L. Goldberg, A. E. Reynolds, L. M. Silver and R. C. Veres, Genetics: From Genes to Genomes, 3rd edn. (Mc Graw Hill, 2008).

13. Š. Hošková, J. Chvalina and P. Račková, Transposition hypergroups of Fredholm integral operators and related hyperstructures. Part I, J.Basic Science, 4 (2008), 43-54.

14. Š. Hošková, J. Chvalina and P. Račková, Transposition hypergroups of Fredholm integral operators and related hyperstructures. Part II, J. Basic Science, 4 (2008), 55-60.

15. J. Jantosciak, Homomorphisms, equivalences and reductions in hypergroups, Riv. Mat. Pura Appl., 9 (1991), 23-47.

16. J. Jantosciak, Transposition hypergroups: Noncommutative join spaces, J. Algebra, 187 (1997), 97-119.

17. F. Marty, Sur une generalization de la notion de group, In: 8th Congress Math. Scandenaves, (1934), pp. 45-49.

18. G. Mendel, Versuche uber Pflanzen-Hybriden, Verh Naturforsheh. Ver. Brunn 4(1866) 3-47 (in English in: J. R. Hortic. Soc. 26 (1901) 1-32.

19. J. Mittas, Hypergroups canoniques, Math. Balkanica, 2 (1972), 165-179.

20. B. A. Pierce, Genetics, A conceptual approach, 4th edition, South Western University, New York.

21. W. Prenowitz, Spherical geometries and multigroups, Canadian J. Math., 2 (1950), 100-119.

22. W. Prenowitz, A contemporary approach to classical geometry, Amer. Math. Monthly, 68 (1961), part II v+67 pp.

23. W. Prenowitz and J. Jantosciak, Join Geometries, Springer-Verlag, UTM., 1979.

24. S. Spartalis, A. Dramalides, and T. Vougiouklis. On $H_{v}$-group rings, Algebras Groups and Geometries, 15 (1988), 47-54. 
25. T. Vougiouklis, Cyclicity in a special class of hypergroups, Acta Univ. Carolin. Math. Phys. 22(1) (1981), 3-6.

26. T. Vougiouklis, Hyperstructures and their Representations, Hadronic Press, Inc 115 Palm Harber, USA, 1994.

27. T. Vougiouklis, Convolutions on WASS hyperstructures, Discrete Mathematics, 174 (1997), 347-355.

28. T. Vougiouklis, The fundamental relation in hyperrings. The general hyperfield, Algebraic hyperstructures and applications (Xanthi, 1990), 203-211, World Sci. Publ., Teaneck, NJ, 1991.

29. L.A. Zadeh, Fuzzy Sets, Information and Control, 8 (1965), 338-353.

\author{
Madeleine Al-Tahan \\ Lebanese International University \\ Faculty of Science \\ P. O. Box 5 Jeb-jenine \\ Khyara, Lebanon \\ madeline.tahan@liu.edu.lb \\ Bijan Davvaz \\ Yazd University \\ Faculty of Science \\ Department of Mathematics \\ P. O. Box 89195-741 \\ Yazd, Iran \\ davvaz@yazd.ac.ir
}

Department of Mathematics and Physics 